\title{
El valor del francés en el mundo: más allá de la utilidad
}

\author{
Marco Antonio Gallardo URIBE \\ Universidad Nacional Autónoma de México
}

\begin{abstract}
En el marco del coloquio Pluralidad de las Lenguas-culturas, en la mesa redonda intitulada “¿Por qué estudiar letras y lenguas modernas?”, se expone la diferencia entre la utilidad y el valor en el aprendizaje de la lengua y las letras modernas (francesas).
\end{abstract}

PALABRAS ClAVE: francés, lengua, letras, valor, utilidad, experiencia, Ernesto de la Peña, UNAM.

Within the framework of the colloquium Plurality of Languages-Cultures, in the panel of discussion called "Why study modern literature?", the difference between the utility and the value of learning modern language and literature, specifically french, is exposed.

KEY WORDS: french, language, literature, value, utility, experience, Ernesto de la Peña, UNAM.

Quisiera comenzar planteando un par de enunciados tal vez un tanto polémicos. A muchos se nos ha cuestionado el haber elegido estudiar letras en lugar de haber optado por alguna otra carrera más "útil” y más "productiva" para la sociedad. En general, en nuestro país, aventurarse a estudiar letras es casi equivalente a "morirse de hambre" y a no encontrar un empleo fijo o acaso a "ser profesor toda la vida". Aclaro que no menosprecio el trabajo docente, me parece uno de los más importantes para todo país - para toda comunidad - cuando se realiza con el compromiso y la seriedad que requiere, cuando auténticos maestros son los encargados de la enseñanza, pero sí resalto que no se suele considerar el resto de las actividades que podemos llevar a cabo ni la importancia y el impacto que pueden ejercer en el desarrollo individual y social. Menciono lo anterior no porque esté de acuerdo con aquella postura - la del instrumento de un modelo que domina infundiendo "miedo al futuro, miedo al desempleo, miedo a un mundo sin rumbo" (González: 117)—, sino porque, primeramente, me parece que esa concepción es errónea y refleja dos problemas de fondo mucho más graves que están enraizados en la mentalidad de los mexicanos: la falta de crítica y reflexión y la falta de apertura a otras formas de pensar. Además, lo traje a colación porque si bien ésa ha sido una opinión común entre la sociedad mexicana, en esta ocasión la misma postura ha sido adoptada por otro actor que solía ser el contrapeso 
de todos aquellos prejuicios. Sin duda alguna, el hecho de que en estos últimos dos días nos hayamos volcado a la reflexión sobre el quehacer de todos nosotros, estudiantes y profesores, muestra el interés que tenemos en pensar y reflexionar. Sin embargo, esta reflexión no surgió como una meditación de las letras sobre las letras mismas, sino que se alzó como una reacción ante la decisión de relegar el francés a ser una materia extracurricular en el bachillerato de la UNAM. Si bien éstas no son las condiciones en las que me hubiera gustado hablar sobre la lengua y las letras francesas, aprovecharé la ocasión para emprender una apología del campo de estudio que he elegido e intentaré exponerles las razones que hacen, no solamente útil la enseñanza de diversas lenguas en las preparatorias - y por su puesto en nuestra Universidad-, sino que además mostraré que el aprendizaje de los idiomas es valioso en sí mismo y necesario para cualquier sociedad, sobre todo para una como la nuestra, ávida de transformaciones, ansiosa de mejorar, anhelante de criticar, de asombrarse, de imaginar y de crear.

Antes de exponer algunos de los argumentos a favor de la enseñanza de otros idiomas, quisiera aclarar que si a lo largo de esta intervención hablaré específicamente del francés, se debe simplemente a razones prácticas: primeramente porque es la lengua, además del español y del inglés, que más conozco, la que más utilizo y la que más he estudiado; y por otro lado, porque en este momento soy el portavoz de mis compañeros de Letras Francesas. Sin embargo, la mayoría de las razones que expondré más adelante deberán entenderse y aplicarse no sólo a las lenguas que los colegas a mi lado representan, sino también a todas las demás que no se enseñan ni se estudian en nuestra Universidad. Permítanme, pues, empezar con el aspecto estrictamente utilitarista de la enseñanza y del estudio del francés. De antemano ofrezco una disculpa si el listado de cifras que estoy a punto de hacer les parece demasiado frío y técnico, pero considero pertinente desplegar las siguientes estadísticas que parecen ser las únicas que a nuestras autoridades les interesan. Según la Organización Internacional de la Francofonía, al menos existen doscientos veinte millones de francófonos en el mundo y alrededor de ciento dieciséis millones de estudiantes de francés. A dicha organización pertenecen cincuenta y seis Estados miembros y catorce observadores. Además, el francés es la lengua oficial en treinta y dos países (Organistaion Internationale de la Francophonie). El francés, junto con el inglés, es el único idioma que se habla en los cinco continentes (Ministerio de Asuntos Exteriores). A pesar de que por el número de hablantes se sitúa en decimotercer lugar a nivel mundial — después del mandarín, del español, del inglés, del hindi, del árabe, del portugués, del ruso, del bengalí, del japonés, del alemán, del punjabi y del javanés-, el francés continúa siendo la segunda lengua más influyente en el mundo (Weber). El francés es el idioma de trabajo de diversos organismos internacionales como la Organización de las Naciones Unidas, la Unión Europea, la UNESCO, el Comité Olímpico Internacional, la FIFA, la Cruz Roja Internacional, la Organización del Tratado del Atlántico Norte, la Agencia Espacial Europea, el Consejo Europeo para la Investigación Nuclear, la Corte Internacional de Justicia, el Tribunal de Justicia de la Unión Europea, la Organización para la Cooperación y el 
Desarrollo Económico, la Organización Mundial de Comercio y de organizaciones como Médicos sin Fronteras o Reporteros sin Fronteras (Francophonu). En internet es la sexta lengua más utilizada después del inglés, del chino, del español, del japonés y del alemán (Weber). En el caso específico de Francia, ésta es la quinta potencia económica del mundo, es el sexto socio comercial de México entre los países de la UE y México es su segundo socio comercial en América Latina (Secretaría de Relaciones Exteriores). Además, los intercambios comerciales entre ambos países han presentado incrementos representativos a lo largo de los últimos años. Existen alrededor de cuatrocientas empresas francesas en México (Embajada de Francia en México), entre las que se encuentran Peugeot, Renault, Danone, Alcatel, L'Oréal o Air France-KLM, que se traducen en una derrama de cuatro mil millones de dólares anuales y que generan setenta y cinco mil empleos directos (Secretaría de Relaciones Exteriores). Incluso existe el Grupo de Alto Nivel Franco-Mexicano, que tiene como objetivo impulsar las relaciones bilaterales entre ambas naciones (Meyer). Por otro lado, la cooperación entre Francia y México se extiende al campo cultural, de las ciencias y de la tecnología gracias a la fuerte presencia del Instituto Francés para América Latina, de las vientisiete Alianzas Francesas, de la Casa de Francia, del Liceo Franco-Mexicano y del Instituto de Investigación para el Desarrollo, sólo por mencionar algunas instituciones (Embajada de Francia en México). Es más que evidente que el aprendizaje del francés es útil y no solamente para los que estudiamos literatura. Significa un área enorme de posibilidades para los médicos, economistas, politólogos, abogados, físicos, químicos, ingenieros, arquitectos, diseñadores y para todos los profesionales que esta Universidad forma generación con generación. Primeramente porque les abre las puertas para poder estudiar en el extranjero: Francia es el tercer país a nivel mundial en recibir estudiantes de intercambio, alrededor de dos mil seiscientos mexicanos anualmente (Embajada de Francia en México). Además, después de terminar la formación académica, saber francés se traduce en una ventaja competitiva en el mercado laboral internacional, mejorando la expectativa salarial y el nivel de vida de los jóvenes mexicanos.

Queda claro que si analizamos únicamente el aspecto utilitario del francés, éste se convierte en una herramienta poderosa que puede abrir muchos caminos para el desarrollo profesional y material de quienes lo estudian. Sin embargo, lo que a mí más me interesa no es la utilidad del francés sino su valor intrínseco. Aprender otro idioma no solamente implica aprender a pronunciar de otra manera, aprender otro vocabulario y memorizar reglas gramaticales y sintácticas, sino que involucra sumergirse, aprender y entender una cultura diferente, una cosmovisión distinta, una forma de pensar que en ocasiones diverge de nuestra propia concepción del mundo. Si nos situamos en los lugares más comunes, podría decirles que en francés se expresaron grandes escritores como Victor Hugo o Marcel Proust, grandes artistas como Matisse, Monet, Jacques Brel y Edith Piaf, o que fue gracias a su espíritu crítico que grandes filósofos como Sartre, Foucault o Derrida expusieron sus ideas o que fue la lengua de la Ilustración y de los derechos del hombre plasmados por la pluma de personalidades como Voltaire, Rousseau o Montesquieu. En suma, podría decir que el francés es la lengua de la cultura, 
sin embargo, en esta ocasión quisiera centrarme, no en la cultura como una manifestación artística o intelectual, sino en la cultura como la experiencia humana en su totalidad y en el francés en específico como una manifestación concreta de esa experiencia humana. Aprender francés es, para mí, una experiencia, la experiencia de sí mismo, de los otros y la experiencia de la existencia en el mundo. ¿A qué me refiero con experiencia? Es sencillo aprender francés; en lo personal, ello me permitió, en primer lugar, poder pensar de una manera diferente sobre mí mismo, sobre mi país, sobre mi cultura; en segundo lugar, pude acceder a otros puntos de vista, a otras formas de pensar, a otras formas de vivir, a otras formas de actuar, y en tercer lugar, después de verme desde otro punto de vista y ver a los otros desde diferentes perspectivas, me permitió poder dejar de lado las diferencias, para poder coexistir en la diversidad y entender que existe algo más universal: la pertenencia a la humanidad. Permítanme desarrollar brevemente las tres ideas anteriores.

Primero. Todo lenguaje conlleva un proceso de construcción identitaria, en algunos casos más profundo que en otros. Al aprender otro idioma, accedemos a otra forma de estructurar el pensamiento, a otra visión del mundo que permite colocarnos en una posición diferente a la habitual y permite poder mirarnos desde otra perspectiva. Por un lado, esto alienta la crítica personal y también motiva, por lo general, cambios en la manera acostumbrada de acercarse a la realidad inmediata. Les pongo un par de ejemplos: al aprender francés, uno se hace más consciente de su propia lengua, del español, y así lo enriquecemos y lo sofisticamos; al aprender otra manera de pensar, los horizontes y paradigmas en los que vivimos se expanden y se modifican, y podemos ver que las cosas pueden ser de otra manera, que nosotros podemos ser de otra manera. Conocerse, en este sentido, es como volver a nacer.

Segundo. El acceso a otros lenguajes no solamente permite construir una identidad enriquecida y diversa, sino que permite acercarse a otros seres humanos. En el caso específico del francés, si hacemos caso a las cifras antes mencionadas, esto significaría tener la posibilidad de comunicarse con al menos trescientos treinta y seis millones de seres humanos más en el mundo. Es decir, el lenguaje se convierte en un lugar de encuentro, de contacto, de intercambio y de diálogo con los otros. Permite acercarse un poco más a la humanidad. Permite el intercambio de ideas por un lado, pero también de otras experiencias de vida que a su vez enriquecen la existencia propia. Si no impulsáramos el estudio de otras lenguas sería imposible, por ejemplo, acceder a textos en otros idiomas gracias a la enorme labor de los traductores o en todo caso accederíamos únicamente a un puñado de ideas y de seres que se expresan en una sola lengua.

Tercero. Finalmente, además de ser un medio para la construcción personal y un puente para comunicarse con los otros y tener acceso a otras ideas, el aprendizaje de otras lenguas nos permite entender que, a pesar de las diferencias, hay rasgos y actitudes comunes a todos los individuos, es decir, nos permite entender que somos seres únicos que comparten una misma pertenencia, la pertenencia al género humano, a "la aventura humana" (Maalouf: 188). 
Éste es justamente para mí el valor intrínseco de estudiar lengua y letras modernas, que va más allá del mero valor de uso, de la "utilidad", es decir, que permite el acercamiento entre los hombres y en consecuencia la construcción de un mundo mejor, mucho más humano. Esto se promueve al estudiar cualquier idioma, pero no es suficiente con estudiar uno solo con la única finalidad de responder a una demanda del mercado. Que quede claro, no estoy diciendo que debería dejarse de enseñar el inglés - puesto que efectivamente es muy útil—y que sólo debería enseñarse el francés, simplemente quiero decir que la labor de la Universidad no debería apuntar hacia el monolingüismo que empobrece, que se convierte finalmente en una discapacidad para la humanidad, sino que nuestra Universidad debería apostar por la formación de seres humanos libres, reflexivos, analíticos y responsables. En México ya existen muchas instituciones que se dedican a manufacturar estudiantes-producto para el mercado. La UNAM tendría que esforzarse, no en buscar eliminar la enseñanza del francés en las preparatorias, sino en promover su estudio - iy el de la mayor cantidad de lenguas! para ampliar así las posibilidades de elección y en consecuencia de libertad y de responsabilidad. Debe esforzarse por mostrar el rostro humano de la educación contemporánea (González: 111). Además, en lugar de reducir el campo laboral de los que nos aventuramos a estudiar Letras, debería promover la formación de profesores en el seno mismo de nuestra Facultad; debería impulsar la creación de centros de investigación literaria o centros de traducción literaria y especializada; debería también establecer vínculos con diferentes instituciones y organismos para que las nuevas generaciones de profesores, traductores, críticos literarios, editores y escritores podamos realizar nuestro servicio social, para que podamos estudiar en el extranjero y para que podamos acceder a fuentes de trabajo que estén relacionadas con lo que hemos decidido estudiar y que resulta de un gran valor para la humanidad entera.

La UNAM debería seguir defendiendo los ideales humanistas como lo hizo el gran maestro Ernesto de la Peña, ex alumno de esta misma facultad, quien cultivó un interés no sólo por una lengua, sino por más de treinta idiomas. La UNAM no debería supeditar las acciones educativas a criterios mercantilistas, la dignidad de las personas al dominio del mercado y no debería aceptar pasivamente convertirse en un apéndice de éste, aceptando el rol de un simple sirviente y no el de eje rector que coloque al ser humano en un lugar privilegiado (González: 117). Es momento de hacer honor al lema de esta Universidad y permitir que por nuestra raza hable el espíritu, pero que hable el idioma universal de la humanidad expresada en una enorme diversidad de lenguas.

\section{Obras citadas}

Embajada de Francia en MÉXico. 2013. Le Mexique et la France en chiffres. 7 de mayo de 2013. <http://ambafrance-mx.org/Mexico-y-Francia-en-cifras>. Francophonu. 2011. Langue Française. 23 de agosto de 2012. <http://www. francophonu.org $/$ ?article $=36>$. 
GonzÁLEZ, Rafael. 2012. "Entrevista a Carlos de la Isla: el rostro humano en la educación contemporánea”. Estudios. México: ITAM. Pp. 111-134.

MaAlouf, Amin. 2011. Les identités meurtrières. París: Le livre de poche.

MEYER, Jean. 2009. "México y Francia”. El Universal. 7 de mayo de 2013. <http:// www.eluniversal.com.mx/editoriales/43281.html>.

Ministerio de los Asuntos EXTERIORES FrANCÉS. 2012. La lengua francesa en el mundo. 7 de mayo de 2013. <http://www.diplomatie.gouv.fr/es/asuntosglobales/francofonia-y-lengua-francesa/lengua-francesa/la-lengua-francesaen-el-mundo/>.

Organisation Internationale de la Francophonie. 2012. Présentation. 20 de septiembre de 2012. <http://www.francophonie.org>.

SECRETARÍA DE RELACIONES EXTERIORES. 2011. Relaciones Económicas. Embajada de México en Francia. 7 de mayo 2013. <http://embamex.sre.gob.mx/francia/index.php/es/relaciones-economicas>.

WeBER, George. 2008. The World's 10 most influencial Languages. The Adaman Association. 7 de mayo de 2013. <http://www.andaman.org/BOOK/reprints/ weber/rep-weber.htm>. 